\title{
Hearing Loss Detection Based on Wavelet Entropy and Genetic Algorithm
}

\author{
Fangyuan $\mathrm{Liu}^{1}$, Arifur Nayeem ${ }^{2,3}$ and Atiena Pereira ${ }^{4, *}$ \\ ${ }^{1}$ School of Computer Science and Engineering, Nanjing Normal University, Nanjing 210023, P R China \\ ${ }^{2}$ Saidpur government Technical School and College, Saidpur 5310, Bangladesh \\ ${ }^{3}$ School of Overseas Education Nanjing University of Post and Telecommunications, Nanjing, 210046, China \\ ${ }^{4}$ School of Biology, University of Campinas, Campinas, São Paulo, Brazil \\ ${ }^{*}$ Corresponding author
}

\begin{abstract}
In order to develop a new hearing loss detection method, this paper proposed to combine wavelet entropy with feedforward neural network trained by genetic algorithm. The dataset contains 72 subjects-24 healthy controls, 24 left-sided hearing loss patients, and 24 right-sided hearing loss patients. The 10 runs of 8-fold cross validation showed that optimal decomposition level was 4 , better than the results using decomposition level of 2,3 , and 5 . Our method using 4-level decomposition yielded a sensitivity for healthy controls of $81.25 \pm 4.91 \%$, a sensitivity for left-sided hearing loss of $\mathbf{8 0 . 4 2} \pm 5.57 \%$, a sensitivity for right-sided hearing loss of $81.67 \pm 6.86 \%$, and an overall accuracy of $81.11 \pm 1.34 \%$.
\end{abstract}

Keywords-hearing loss; wavelet entropy; feedforward neural network; genetic algorithm

\section{INTRODUCTION}

Hearing loss (HL) [1] may occur in one of both ears. The HL problems in children may even destroy his/her spoken ability. It may cause loneliness for adults. Nowadays scholars prefer to use magnetic resonance imaging (MRI) [2-4] method to detect hearing loss.

For example: Li [5] proposed a fractional Fourier transform method. Jia [6] used deep autoencoder method. Liu [7] suggested to use dual-tree complex wavelet transform. Li [8] offered a new method using fitness-scaling adaptive genetic algorithm. Nayak [9] used stationary wavelet transform and Shannon entropy. Chen [10] gave a new method of using generalized eigenvalue proximal support vector machine. Gorriz [11] employed directed acyclic graph support vector machine.

Nevertheless, those methods are too complicated and hard to implement. In addition, their methods may not get accurate results that meet practical requirement. Further, some algorithms are too time-consuming.

Our contribution is we proposed a novel method that combined wavelet entropy, feedforward neural network, and genetic algorithm. Our method shows promising results in identifying hearing loss patients.

\section{SUBJECTS}

We collected 72 magnetic resonance brain images from local hospitals. The dataset can be divided into three categories, including 24 healthy brain images, 24 left HL brain images, and 24 right HL brain images. Written consents were obtained from

all subjects. Different types of hearing loss images are shown below in Figure 1.

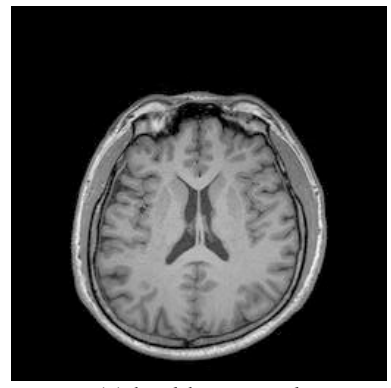

(a) healthy control

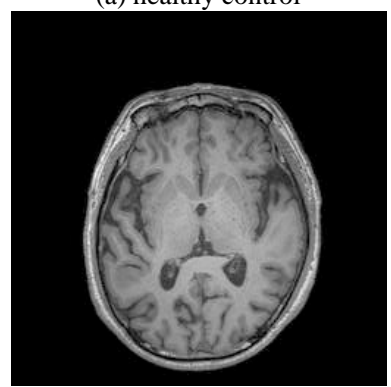

(c) right-sided hearing loss

FIGURE I. SAMPLE OF BRAIN IMAGES

\section{METHODOLOGY}

Wavelet entropy is a novel method to analyze transient features of complicated images. It has already been applied in satellite image processing, brain image processing [12-17], face recognition $[18,19]$, etc. The pseudocode of wavelet entropy is listed in Table 1.

TABLE I. ALGORITHM OF WAVELET ENTROPY

\section{Algorithm - Wavelet entropy (WE)}

Step A Import the magnetic resonance brain image;

Step B Choose the wavelet family;

Step C Choose decomposition level $k$;

Step D Perform discrete wavelet transform (DWT) on given brain image;

Step E Generate and record $(3 k+1)$ wavelet subbands;

Step F Calculate entropy over each subband;

Step G Vectorize all the entropy results and output it as the feature 


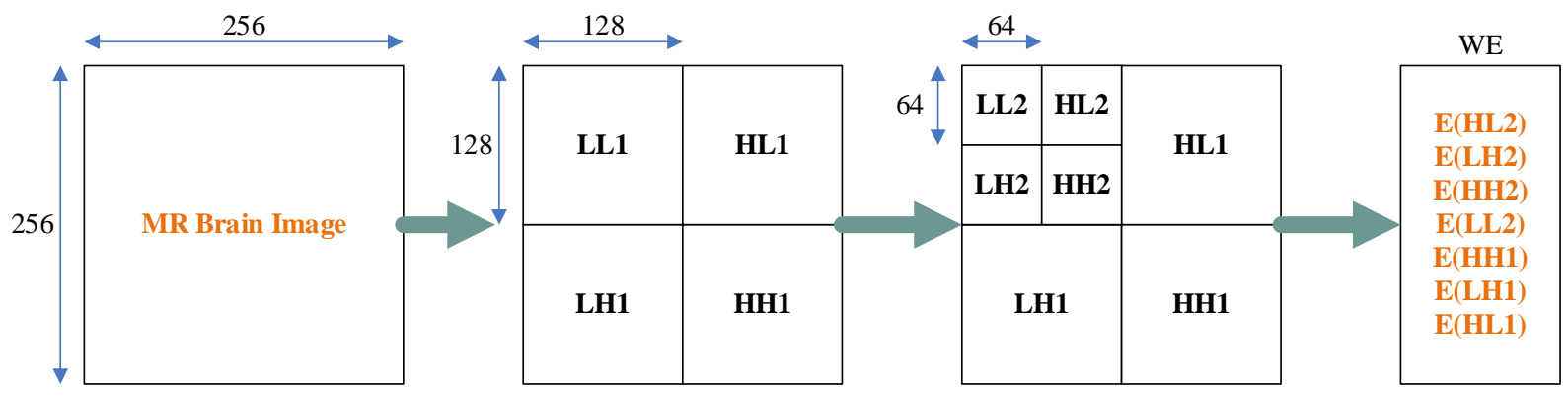

FIGURE II. DIAGRAM OF CARRYING OUT WAVELET ENTROPY

Figure 2 shows a diagram of carrying out a 2-level wavelet entropy. Here we first decompose 1-level discrete wavelet transform (DWT) over the magnetic resonance (MR) brain image, and obtained four subbands (LL1, LH1, HL1, and HH1) [20]. Then, we used DWT to decompose the LL1, and obtained four new subbands (LL2, LH2, HL2, and HH2). In total, we get 7 subbands altogether. Finally, entropy was performed over these seven subbands.

In this study, we chose bior5.5 wavelet as suggested in Ref. [11]. Here a "bior $x . y$ " means a B-spline biorthogonal compactly supported wavelet with reconstruction order of $x$ and decomposition order of $y$. The decomposition functions and filters of bior5.5 wavelet are shown in Figure 3.

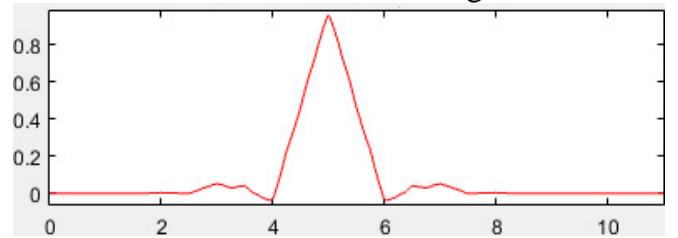

(a) Scaling Function

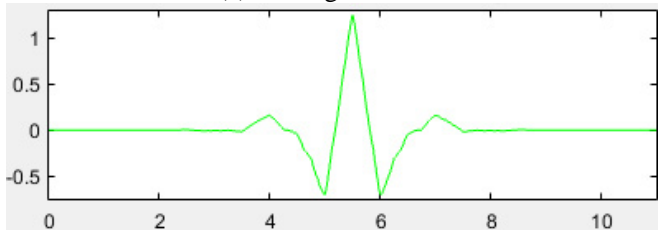

(b) Wavelet Function

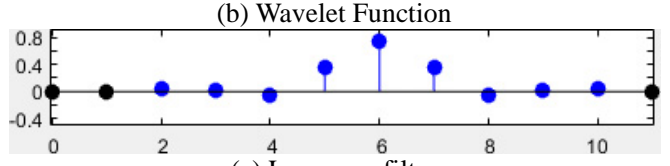

(c) Low-pass filter

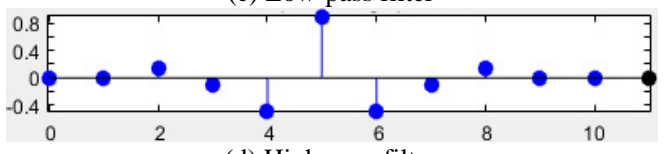

(d) High-pass filter

FIGURE III. DECOMPOSITION FOR BIOR5.5

The features extracted by WE was submitted to a feedforward neural network (FNN), also named multilayer perceptron [21]. It does not need any a priori information about the data distribution. Scholars have reported that FNN gained remarkable success compared to traditional classifiers [22-26]. Suppose it contains $N_{I}$ input nodes, $N_{H}$ hidden nodes, and $N_{O}$ output nodes. We can draw its fully-connected structure in Figure 4. We did not use deep learning methods, such as convolutional neural network [27-30] and autoencoder [31, 32], because our dataset in this study is relatively small.

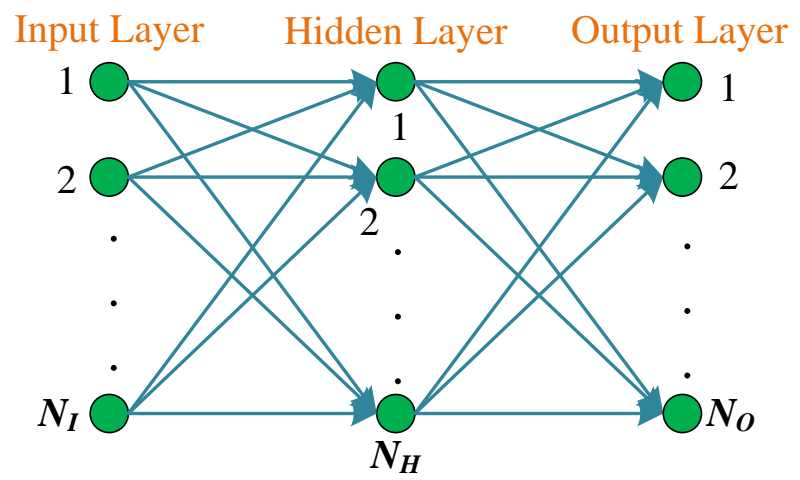

FIGURE IV. STRUCTURE OF FNN

Finally, traditional back propagation algorithm was an FNN training method. Nevertheless, it may fall into local optimum. Hence, to avoid this problem, we used genetic algorithm (GA) to train the FNN. We first transform the training to an optimization problem using mean squared error (MSE) [33-36] as fitness function.

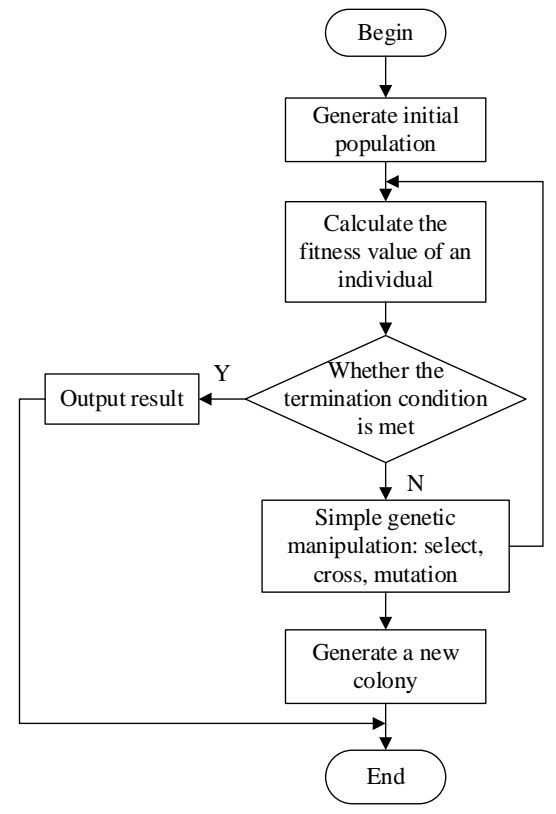

FIGURE V. DIAGRAM OF GENETIC ALGORITHM 
Then in a standard GA, each candidate solution is named as the chromosome [37, 38], and the whole population evolved towards better solution by three implementations: crossover, mutation, and selection. Figure 5 shows the diagram of a standard GA. Detailed description can be found in Ref. [39-41].

\section{EXPERIMENTS AND RESULTS}

Eight-fold cross validation was used. In each fold, we contains three healthy controls, three left-sided HL brain images, and three right-sided HL brain images. The optimal decomposition level was found to be 4 . The statistical results of 10 runs of eight-fold cross validation are listed in Table 2.

TABLE II. CORRECTLY IDENTIFIED RESULTS ( $\mathrm{F}=$ FOLD; $\mathrm{R}=\mathrm{RUN})$

\begin{tabular}{|l|l|l|l|l|l|l|l|l|l|}
\hline & $\mathrm{F} 1$ & $\mathrm{~F} 2$ & $\mathrm{~F} 3$ & $\mathrm{~F} 4$ & $\mathrm{~F} 5$ & $\mathrm{~F} 6$ & $\mathrm{~F} 7$ & $\mathrm{~F} 8$ & Total \\
\hline $\mathrm{R}$ & $3+3$ & $3+2$ & $2+2$ & $2+3$ & $3+2$ & $2+2$ & $2+3$ & $3+3$ & $20+20$ \\
1 & +3 & +2 & +3 & +2 & +3 & +2 & +3 & +0 & $\begin{array}{l}+18 \\
\end{array}$ \\
$=9$ & $=7$ & $=7$ & $=7$ & $=8$ & $=6$ & $=8$ & $=6$ & $=58$ \\
\hline \multirow{2}{*}{$\mathrm{R}$} & $3+2$ & $3+2$ & $1+3$ & $3+2$ & $2+2$ & $3+2$ & $2+2$ & $2+3$ & $19+18$ \\
2 & +3 & +3 & +3 & +3 & +1 & +3 & +3 & +3 & +22 \\
& $=8$ & $=8$ & $=7$ & $=8$ & $=5$ & $=8$ & $=7$ & $=8$ & $=59$ \\
\hline $\mathrm{R}$ & $3+3$ & $3+3$ & $2+3$ & $1+2$ & $3+3$ & $2+3$ & $3+1$ & $3+3$ & $20+21$ \\
3 & +2 & +1 & +3 & +3 & +3 & +2 & +2 & +3 & +19 \\
& $=8$ & $=7$ & $=8$ & $=6$ & $=9$ & $=7$ & $=6$ & $=9$ & $=60$ \\
\hline $\mathrm{R}$ & $3+1$ & $3+3$ & $2+3$ & $3+2$ & $2+3$ & $0+3$ & $3+1$ & $2+3$ & $18+19$ \\
4 & +3 & +2 & +2 & +3 & +3 & +3 & +3 & +3 & +22 \\
& $=7$ & $=8$ & $=7$ & $=8$ & $=8$ & $=6$ & $=7$ & $=8$ & $=59$ \\
\hline $\mathrm{R}$ & $1+2$ & $3+3$ & $3+2$ & $2+2$ & $3+2$ & $3+3$ & $2+3$ & $3+3$ & $20+20$ \\
5 & +3 & +2 & +2 & +2 & +1 & +3 & +3 & +3 & +19 \\
& $=6$ & $=8$ & $=7$ & $=6$ & $=6$ & $=9$ & $=8$ & $=9$ & $=59$ \\
\hline $\mathrm{R}$ & $3+3$ & $3+1$ & $1+3$ & $2+3$ & $3+1$ & $3+2$ & $3+3$ & $3+3$ & $21+19$ \\
6 & +2 & +3 & +3 & +3 & +3 & +3 & +0 & +2 & +19 \\
& $=8$ & $=7$ & $=7$ & $=8$ & $=7$ & $=8$ & $=6$ & $=8$ & $=59$ \\
\hline \multirow{2}{*}{$\mathrm{R}$} & $3+3$ & $2+2$ & $2+3$ & $3+1$ & $2+2$ & $1+3$ & $2+3$ & $2+3$ & $17+20$ \\
7 & +3 & +3 & +2 & +2 & +3 & +2 & +3 & +2 & +20 \\
& $=9$ & $=7$ & $=7$ & $=6$ & $=7$ & $=6$ & $=8$ & $=7$ & $=57$ \\
\hline \multirow{2}{*}{$\mathrm{R}$} & $3+2$ & $1+3$ & $3+2$ & $3+3$ & $3+1$ & $2+1$ & $3+3$ & $2+3$ & $20+18$ \\
8 & +2 & +3 & +2 & +2 & +1 & +3 & +3 & +3 & +19 \\
& $=7$ & $=7$ & $=7$ & $=8$ & $=5$ & $=6$ & $=9$ & $=8$ & $=57$ \\
\hline \multirow{2}{*}{$\mathrm{R}$} & $3+3$ & $3+2$ & $3+3$ & $2+3$ & $2+3$ & $3+3$ & $1+2$ & $3+2$ & $20+21$ \\
9 & +2 & +2 & +3 & +2 & +1 & +3 & +2 & +2 & +17 \\
& $=8$ & $=7$ & $=9$ & $=7$ & $=6$ & $=9$ & $=5$ & $=7$ & $=58$ \\
\hline \multirow{2}{*}{$\mathrm{R}$} & $2+2$ & $2+2$ & $2+2$ & $3+2$ & $2+3$ & $3+3$ & $\begin{array}{l}3+2 \\
+3\end{array}$ & $3+1$ & $20+17$ \\
10 & +3 & +3 & +3 & +1 & +3 & +3 & +3 & +2 & +21 \\
& $=7$ & $=7$ & $=7$ & $=6$ & $=8$ & $=9$ & $=8$ & $=6$ & $=58$ \\
\hline
\end{tabular}

Here $(a+b+c)=z$ means $a$ healthy controls, $b$ left-sided, and $c$ right-sided were correctly identified. In total $z$ brains were identified.
TABLE III. SENSITIVITY OF THREE CLASSES OF 10 RUNS (DECOMPOSITION LEVEL $=4$ )

\begin{tabular}{|l|l|l|l|l|}
\hline Run & Healthy & Left & Right & Overall \\
\hline R1 & 83.33 & 83.33 & 75.00 & 80.56 \\
\hline R2 & 79.17 & 75.00 & 91.67 & 81.94 \\
\hline R3 & 83.33 & 87.50 & 79.17 & 83.33 \\
\hline R4 & 75.00 & 79.17 & 91.67 & 81.94 \\
\hline R5 & 83.33 & 83.33 & 79.17 & 81.94 \\
\hline R6 & 87.50 & 79.17 & 79.17 & 81.94 \\
\hline R7 & 70.83 & 83.33 & 83.33 & 79.17 \\
\hline R8 & 83.33 & 75.00 & 79.17 & 79.17 \\
\hline R9 & 83.33 & 87.50 & 70.83 & 80.56 \\
\hline R10 & 83.33 & 70.83 & 87.50 & 80.56 \\
\hline Avr & $81.25 \pm 4.91$ & $80.42 \pm 5.57$ & $81.67 \pm 6.86$ & $81.11 \pm 1.34$ \\
\hline
\end{tabular}

The sensitivities of each classes are shown in Table 3. Here our method yields a sensitivity for healthy controls of $81.25 \pm 4.91 \%$, a sensitivity for left-sided hearing loss of $80.42 \pm 5.57 \%$, a sensitivity for right-sided hearing loss of $81.67 \pm 6.86 \%$, and an overall accuracy of $81.11 \pm 1.34 \%$.

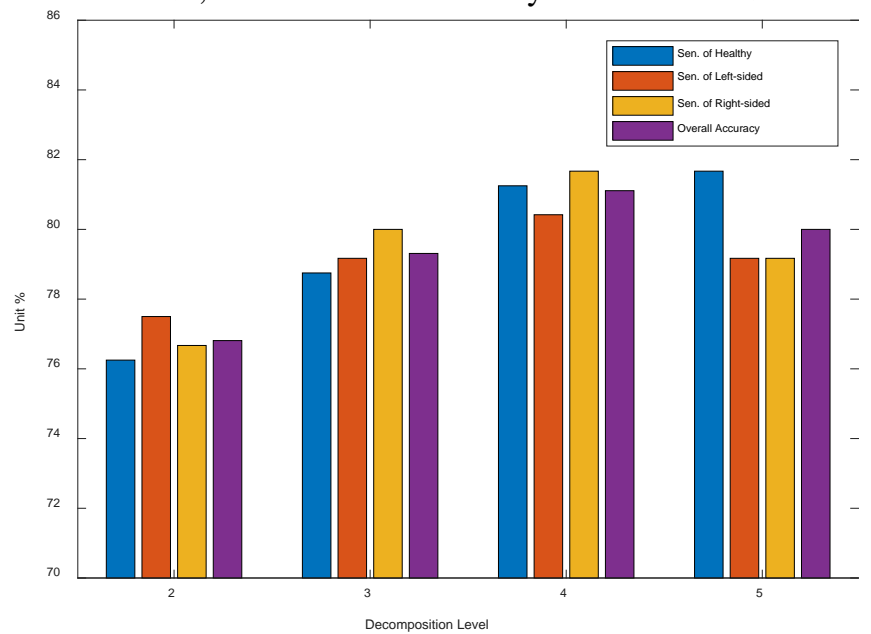

FIGURE VI. OPTIMAL LEVEL

TABLE IV. SENSITIVITY OF THREE CLASSES OF 10 RUNS (DECOMPOSITION LEVEL = 2, 3, AND 5)

\begin{tabular}{|l|l|l|l|l|l|l|l|l|l|l|l|l|}
\hline \multirow{2}{*}{ Run } & \multicolumn{3}{|l|}{ Decomposition Leve = 2 } & \multicolumn{3}{|l|}{ Decomposition Level = 3 } & \multicolumn{3}{l|}{ Decomposition Level = 5 } \\
\cline { 2 - 15 } & Healthy & Left & Right & Overall & Healthy & Left & Right & Overall & Healthy & Left & Right & Overall \\
\hline R1 & 70.83 & 83.33 & 75.00 & 76.39 & 79.17 & 83.33 & 83.33 & 81.94 & 83.33 & 87.50 & 75.00 & 81.94 \\
\hline R2 & 75.00 & 79.17 & 70.83 & 75.00 & 79.17 & 87.50 & 75.00 & 80.56 & 79.17 & 83.33 & 75.00 & 79.17 \\
\hline R3 & 79.17 & 75.00 & 79.17 & 77.78 & 75.00 & 75.00 & 83.33 & 77.78 & 91.67 & 87.50 & 66.67 & 81.94 \\
\hline R4 & 70.83 & 83.33 & 70.83 & 75.00 & 75.00 & 79.17 & 87.50 & 80.56 & 87.50 & 75.00 & 87.50 & 83.33 \\
\hline R5 & 79.17 & 79.17 & 79.17 & 79.17 & 83.33 & 75.00 & 83.33 & 80.56 & 70.83 & 79.17 & 75.00 & 75.00 \\
\hline R6 & 79.17 & 75.00 & 75.00 & 76.39 & 83.33 & 79.17 & 75.00 & 79.17 & 83.33 & 79.17 & 83.33 & 81.94 \\
\hline R7 & 87.50 & 66.67 & 75.00 & 76.39 & 83.33 & 75.00 & 66.67 & 75.00 & 87.50 & 75.00 & 75.00 & 79.17 \\
\hline R8 & 75.00 & 75.00 & 83.33 & 77.78 & 79.17 & 79.17 & 79.17 & 79.17 & 87.50 & 62.50 & 83.33 & 77.78 \\
\hline R9 & 79.17 & 79.17 & 75.00 & 77.78 & 79.17 & 79.17 & 75.00 & 77.78 & 66.67 & 83.33 & 87.50 & 79.17 \\
\hline R10 & 66.67 & 79.17 & 83.33 & 76.39 & 70.83 & 79.17 & 91.67 & 80.56 & 79.17 & 79.17 & 83.33 & 80.56 \\
\hline \multirow{2}{*}{ Avr } & $76.25 \pm$ & $77.50 \pm$ & $76.67 \pm$ & $76.81 \pm$ & $78.75 \pm$ & $79.17 \pm$ & $80.00 \pm$ & $79.31 \pm$ & $81.67 \pm$ & $79.17 \pm$ & $79.17 \pm$ & $80.00 \pm$ \\
& 5.91 & 4.89 & 4.48 & 1.32 & 4.14 & 3.93 & 7.30 & 2.01 & 7.91 & 7.35 & 6.80 & 2.47 \\
\hline
\end{tabular}


In order to validate why we chose 4-level as the optimal decomposition level, we showed the results using 2, 3, and 5 levels in Table 4. Here it shows the overall accuracy of 2-level decomposition was $76.81 \pm 1.32 \%$, the overall accuracy of 3level decomposition was $79.31 \pm 2.01 \%$, and the overall accuracy of 5-level decomposition was $80.00 \pm 2.47 \%$. Compared to the results of 4-level decomposition, we can see that 4-level obtained the better result than 2-level, 3-level, and 5-level did, as shown in Figure 6.

\section{CONCLUSION}

This study give a new method for hearing loss detection based on wavelet entropy, feedforward neural network, and genetic algorithm. In the future, we shall test our method on a larger dataset of hearing loss diseases.

\section{ACKNOWLEDGEMENT}

The paper is financially supported by Postgraduate Research \& Practice Innovation Program of Jiangsu Province (SJCX17_0342).

\section{REFERENCE}

[1] Mitchell, R.M., B.S. Saltzman, et al., Hearing Loss in Children With Craniofacial Microsomia. Cleft Palate-Craniofacial Journal, 2017. 54(6): pp. 656-663

[2] Yu, D., H. Shui, et al., Exponential wavelet iterative shrinkage thresholding algorithm with random shift for compressed sensing magnetic resonance imaging. IEEJ Transactions on Electrical and Electronic Engineering, 2015. 10(1): pp. 116-117

[3] Yang, J.F. and P. Sun, A Novel Compressed Sensing Method for Magnetic Resonance Imaging: Exponential Wavelet Iterative ShrinkageThresholding Algorithm with Random Shift. International Journal Of Biomedical Imaging, 2016, Article ID: 9416435

[4] Dong, Z. and P. Phillips, Exponential wavelet iterative shrinkage thresholding algorithm for compressed sensing magnetic resonance imaging. Information Sciences, 2015. 322: pp. 115-132

[5] Li, J., Detection of Left-Sided and Right-Sided Hearing Loss via Fractional Fourier Transform. Entropy, 2016. 18(5), Article ID: 194

[6] Jia, W., Three-Category Classification of Magnetic Resonance Hearing Loss Images Based on Deep Autoencoder. Journal of Medical Systems, 2017. 41, Article ID: 165

[7] Liu, B., Preliminary Study on Unilateral Sensorineural Hearing Loss Identification via Dual-Tree Complex Wavelet Transform and Multinomial Logistic Regression, in Natural and Artificial Computation for Biomedicine and Neuroscience, eds. J.M. Ferrández Vicente, et al., 2017, Springer International Publishing: Cham. pp. 289-297.

[8] Li, J., Texture analysis method based on fractional Fourier entropy and fitness-scaling adaptive genetic algorithm for detecting left-sided and right-sided sensorineural hearing loss. Fundamenta Informaticae, 2017. 151(1-4): pp. 505-521

[9] Nayak, D.R., Detection of unilateral hearing loss by Stationary Wavelet Entropy. CNS \& Neurological Disorders - Drug Targets, 2017. 16(2): pp. $122-128$

[10] Chen, Y. and X.-Q. Chen Sensorineural hearing loss detection via discrete wavelet transform and principal component analysis combined with generalized eigenvalue proximal support vector machine and Tikhonov regularization. Multimedia Tools and Applications, 2016, DOI: 10.1007/s11042-016-4087-6 (Online).

[11] Gorriz, J.M. and J. Ramírez, Wavelet entropy and directed acyclic graph support vector machine for detection of patients with unilateral hearing loss in MRI scanning. Frontiers in Computational Neuroscience, 2016. 10, Article ID: 160

[12] Phillips, P. and J. Yang, Pathological brain detection in magnetic resonance imaging scanning by wavelet entropy and hybridization of biogeography-based optimization and particle swarm optimization. Progress In Electromagnetics Research, 2015. 152: pp. 41-58

[13] Sun, P., Pathological brain detection based on wavelet entropy and $\mathrm{Hu}$ moment invariants. Bio-Medical Materials and Engineering, 2015. 26(s1): pp. 1283-1290

[14] Zhou, X.X., Detection of abnormal MR brains based on wavelet entropy and feature selection. IEEJ Transactions on Electrical and Electronic Engineering, 2016. 11(3): pp. 364-373

[15] Wang, S.-H. Single slice based detection for Alzheimer's disease via wavelet entropy and multilayer perceptron trained by biogeography-based optimization. Multimedia Tools and Applications, 2016, DOI: 10.1007/s11042-016-4222-4 (Online).

[16] Sun, J.-D. Multivariate Approach for Alzheimer's disease Detection Using Stationary Wavelet Entropy and Predator-Prey Particle Swarm Optimization. Journal of Alzheimer's Disease, 2017, DOI: 10.3233/JAD170069 (Online).

[17] Atangana, A. Application of stationary wavelet entropy in pathological brain detection. Multimedia Tools and Applications, 2016, DOI: 10.1007/s11042-016-3401-7 (Online).

[18] Lu, H.M., Facial Emotion Recognition Based on Biorthogonal Wavelet Entropy, Fuzzy Support Vector Machine, and Stratified Cross Validation. IEEE Access, 2016. 4: pp. 8375-8385

[19] Phillips, P., Intelligent facial emotion recognition based on stationary wavelet entropy and Jaya algorithm. Neurocomputing, 2017. 272: pp. 668-676

[20] Zhan, T.M. and Y. Chen, Multiple Sclerosis Detection Based on Biorthogonal Wavelet Transform, RBF Kernel Principal Component Analysis, and Logistic Regression. IEEE Access, 2016. 4: pp. 7567-7576

[21] Sun, Y., A Multilayer Perceptron Based Smart Pathological Brain Detection System by Fractional Fourier Entropy. Journal of Medical Systems, 2016. 40(7), Article ID: 173

[22] Ji, G., Fruit classification using computer vision and feedforward neural network. Journal of Food Engineering, 2014. 143: pp. 167-177

[23] Wei, L., Fruit classification by wavelet-entropy and feedforward neural network trained by fitness-scaled chaotic $\mathrm{ABC}$ and biogeography-based optimization. Entropy, 2015. 17(8): pp. 5711-5728

[24] Wu, J., Fruit classification by biogeography-based optimization and feedforward neural network. Expert Systems, 2016. 33(3): pp. 239-253

[25] Feng, C., Feed-forward neural network optimized by hybridization of PSO and ABC for abnormal brain detection. International Journal of Imaging Systems and Technology, 2015. 25(2): pp. 153-164

[26] Rao, R.V. and A. Liu, Abnormal Breast Detection in Mammogram Images by Feed-forward Neural Network trained by Jaya Algorithm. Fundamenta Informaticae, 2017. 151(1-4): pp. 191-211

[27] Muhammad, K. Image based fruit category classification by 13-layer deep convolutional neural network and data augmentation. Multimedia Tools and Applications, 2017, DOI: 10.1007/s11042-017-5243-3 (Online).

[28] Sun, J. Polarimetric synthetic aperture radar image segmentation by convolutional neural network using graphical processing units. Journal of Real-Time Image Processing, 2017, DOI: 10.1007/s11554-017-0717-0 (Online).

[29] Jiang, Y., X. Hou, et al., Cerebral Micro-Bleed Detection Based on the Convolution Neural Network With Rank Based Average Pooling. IEEE Access, 2017. 5: pp. 16576-16583

[30] Lv, Y.-D. and Y. Sui Alcoholism detection by data augmentation and convolutional neural network with stochastic pooling. Journal of Medical Systems, 2017, DOI: 10.1007/s10916-017-0845-x (Online).

[31] Jia, W. Five-category classification of pathological brain images based on deep stacked sparse autoencoder. Multimedia Tools and Applications, 2017, DOI: 10.1007/s11042-017-5174-z (Online).

[32] Chen, H. Seven-layer deep neural network based on sparse autoencoder for voxelwise detection of cerebral microbleed. Multimedia Tools and Applications, 2017, DOI: 10.1007/s11042-017-4554-8 (Online).

[33] Lu, S. and X. Qiu, A Pathological Brain Detection System based on Extreme Learning Machine Optimized by Bat Algorithm. CNS \& Neurological Disorders - Drug Targets, 2017. 16(1): pp. 23-29 
[34] Yang, J., Pathological brain detection in MRI scanning via Hu moment invariants and machine learning. Journal of Experimental \& Theoretical Artificial Intelligence, 2017. 29(2): pp. 299-312

[35] Yang, J., Preclinical diagnosis of magnetic resonance (MR) brain images via discrete wavelet packet transform with Tsallis entropy and generalized eigenvalue proximal support vector machine (GEPSVM). Entropy, 2015. 17(4): pp. 1795-1813

[36] Liu, A., Magnetic resonance brain image classification via stationary wavelet transform and generalized eigenvalue proximal support vector machine. Journal of Medical Imaging and Health Informatics, 2015. 5(7): pp. 1395-1403

[37] Wei, L. and J. Yang, Fitness-scaling adaptive genetic algorithm with local search for solving the Multiple Depot Vehicle Routing Problem. SIMULATION, 2016. 92(7): pp. 601-616

[38] Correcher, J.F. and R. Alvarez-Valdes, A biased random-key genetic algorithm for the time-invariant berth allocation and quay crane assignment problem. Expert Systems with Applications, 2017. 89: pp. 112-128

[39] Zhang, Y. and L. Wu. Recursive structure element decomposition using migration fitness scaling genetic algorithm. in 2nd International Conference on Swarm Intelligence. 2011. Chongqing, China: Springer Verlag. pp. 514-521

[40] Ji, G., Genetic Pattern Search and Its Application to Brain Image Classification. Mathematical Problems in Engineering, 2013. 2013, Article ID: 580876

[41] Ji, G.L., A Rule-Based Model for Bankruptcy Prediction Based on an Improved Genetic Ant Colony Algorithm. Mathematical Problems in Engineering, 2013, Article ID: 753251 